\title{
Reply to J. F. McKenzie et al.'s comment on “Obliquely propagating large amplitude solitary waves in charge neutral plasmas"
}

\author{
F. Verheest ${ }^{1,2}$ \\ ${ }^{1}$ Sterrenkundig Observatorium, Universiteit Gent, Krijgslaan 281, 9000 Gent, Belgium \\ ${ }^{2}$ School of Physics, University of KwaZulu-Natal, Private Bag X54001, Durban 4000, South Africa
}

Received: 1 June 2007 - Accepted: 4 August 2007 - Published: 27 August 2007

I note the comments by McKenzie et al. (2007), but it may be wise to remember that the plasma approximation, in which quasi-neutrality holds with $n_{i} \simeq n_{e}$ but $\nabla \cdot \boldsymbol{E} \neq 0$ (Chen, 1974), is precisely that: an approximation. Arguments given by McKenzie et al. (2007), and indeed by several other authors, indicating how reasonable the plasma approximation is, are almost all based on linear wave theory and on keeping the relativistic effects small. In addition, it should be borne in mind that the plasma approximation is true only for lowfrequency motions where the electron inertia is not a factor (Chen, 1974; Nicholson, 1983), and, as has been noted before (Verheest, 2007), the concept of frequency might be borrowed from the linear counterparts of the nonlinear waves studied, but it is not a well defined property of stationary modes studied in their own reference frames.

Although the plasma approximation has been used extensively in linear and small amplitude work, it is a legitimate question to ask what its effects might be for large nonlinear structures. In this context, the step was taken (Verheest, 2007) of considering the extreme case in which $n_{i}=n_{e}$ and $\nabla \cdot \boldsymbol{E}=0$.

An indication of what might happen is given by the reductive perturbation treatment of moderately nonlinear electromagnetic waves propagating parallel to the ambient field. As is well known from many papers since the original derivation by Rogister (1971), these waves are governed by the derivative nonlinear Schrödinger (DNLS) equation. If one starts the description from the full set of Maxwell's equations, including Poisson's equation and the displacement current, one finds that to lowest order the parallel electric field vanishes, by combining the equations of motion to that order and without having assumed charge neutrality or relied upon Poisson's equation (see e.g. Verheest, 2000). Charge neutrality

Correspondence to: F. Verheest

(frank.verheest@ugent.be) then follows to lowest order from Poisson's equation, without having been assumed a priori.

Continuing the iterative procedure, one eliminates the higher-order electromagnetic fields by combining in a judicious way the results obtained from the continuity and momentum equations and plugging these into Ampère's law, to arrive at the DNLS equation governing the perpendicular wave magnetic field. Usually the discussion then focuses on the solutions of the DNLS equation and the higher-order fields are not further investigated. The DNLS equation admits only envelope solitons, where the amplitude of the wave magnetic field shows the profile of a traditional solitary wave, but the phase increases linearly on the slow timescale.

However, once the wave magnetic field obeys the DNLS equation, one can evaluate the higher-order electric fields and finds that a parallel electric field has been generated to that order by the nonlinearities (Verheest, 2004). This subsequently tells us that, although at the linear level there is automatically charge neutrality, this cannot be maintained to higher order. Since the cited treatments are nonrelativistic, it is not the relativistic corrections but the nonlinear effects which are responsible for this ultimate charge nonneutrality.

Hence, when now studying large scale nonlinear phenomena for which it is assumed that $n_{i} \simeq n_{e}$, taking the parallel electric field $E_{x}$ zero follows logically from keeping Poisson's equation in the loop. Although the traditional plasma approximation is not followed, this is nevertheless a far cry from being an arbitrary constraint. In view of the potentially larger nonlinearities encountered when studying solitary waves, one is at least entitled to explore the consequences of that.

Moreover, it is puzzling to read (McKenzie et al., 2007) that in the classic nonlinear wave propagating perpendicular to the ambient magnetic field (Adlam and Allen, 1958) the circumstances are not quasi-charge neutral, and that in fact such an assumption would violate conservation of longitudinal momentum, whereas Adlam and Allen (1958) precisely

Published by Copernicus Publications on behalf of the European Geosciences Union and the American Geophysical Union. 
use quasi-neutrality in the form $\left|n_{i}-n_{e}\right| \ll n_{i}$ (my change of their notation for the electron and ion densities) to arrive at their conclusions.

McKenzie et al. (2007) have presented an estimate of the relative charge imbalance when studying nonlinear phenomena like the whistler oscillitons (Sauer et al., 2002; Dubinin et al., 2003; Verheest et al., 2004) based on linear wave theory. An alternative approach might be to solve the parallel equations of motion and get (Verheest et al., 2004)

$E_{x}=\frac{m_{i}-m_{e}}{e} v_{x} \frac{d v_{x}}{d x} \simeq \frac{m_{i}}{e} v_{x} \frac{d v_{x}}{d x}$,

so that Poisson's equation is rewritten as

$$
\frac{\delta n}{n}=\frac{n_{i}-n_{e}}{n}=\frac{1}{2 \omega_{p i}^{2}} \frac{d^{2} v_{x}^{2}}{d x^{2}} .
$$

Here $\omega_{p i}$ is the local ion plasma frequency and $v_{x}$ is the common parallel velocity of the ions and the electrons, a consequence of assuming charge neutrality and of having mass flux conservation $\left(n_{i} v_{i x}=n_{e} v_{e x} \simeq n v_{x}=n_{0} V\right)$ for nonlinear structures studied in a reference frame in which they appear stationary. Equation (2) indicates that the parallel velocities cannot change drastically inside the nonlinear structure and that hence the deviations from the undisturbed value $V$ cannot be large. The implication is that using the plasma approximation restricts the treatment to sufficiently small amplitudes, for arguments which do not rely upon (non)relativistic effects.

In view of the above, we arrive at a picture that was already mentioned in the conclusions of the earlier paper (Verheest, 2007): "Only when the plasma approximation is reasonably obeyed can the concept of oscillitons be salvaged, and this probably not for too large structures. It is thus hoped that more detailed future observations of large scale nonlinear phenomena in space will allow us to discriminate between oscillitons and large wave packets, by focusing on the time variability (or not) of the phase, since the amplitude or envelope graphs look very similar. Present day measurements are not able to do so."
Edited by: J. Büchner

Reviewed by: M. Leubner and another anonymous referee

\section{References}

Adlam, J. H. and Allen, J. E.: The structure of strong collision-free hydromagnetic waves, Philos. Mag., 3, 448-455, 1958.

Chen, F. F.: Introduction to plasma physics, Plenum Press, New York, pp.65-66, 1974.

Dubinin, E., Sauer, K., and McKenzie, J. F.: Nonlinear stationary whistler waves and whistler solitons (oscillitons). Exact solutions, J. Plasma Phys., 69, 305-330, 2003.

McKenzie, J. F., Dubinin, E., and Sauer, K.: Comment on "Obliquely propagating large amplitude solitary waves in charge neutral plasmas” by F. Verheest, Nonlin. Processes Geophys., 14, 49-57, 2007, Nonlin. Processes Geophys., 14, 543-544, 2007.

Nicholson, D. R.: Introduction to plasma theory, John Wiley, New York, pp.139-141, 1983.

Rogister, A.: Parallel propagation of nonlinear low-frequency waves in high- $\beta$ plasma, Phys. Fluids, 14, 2733-2739, 1971.

Sauer, K., Dubinin, E., and McKenzie, J. F.: Wave emission by whistler oscillitons: Application to"coherent lion roars", Geophys. Res. Lett., 29, 2226, doi:10.1029/2002GL015771, 2002.

Verheest, F.: Waves in dusty space plasmas, Kluwer Academic Press, Dordrecht, pp.142-146, 2000.

Verheest, F.: Nonlinear evolution equations for parallel electromagnetic multispecies plasma modes: Reductive perturbation theory in the linear wave frame and electric field generation, Physica Scripta, T113, 92-96, 2004.

Verheest, F.: Obliquely propagating large amplitude solitary waves in charge neutral plasmas, Nonlin. Processes Geophys., 14, 4957, 2007, http://www.nonlin-processes-geophys.net/14/49/2007/.

Verheest, F., Cattaert, T., Dubinin, E., Sauer, K., and McKenzie, J. F.: Whistler oscillitons revisited: the role of charge neutrality?, Nonlin. Processes Geophys., 11, 447-452, 2004, http://www.nonlin-processes-geophys.net/11/447/2004/. 